\title{
Laser-nanostructured Ag films as substrates for surface-enhanced Raman spectroscopy
}

\author{
S. J. Henley, ${ }^{\text {a) }}$ J. D. Carey, and S. R. P. Silva \\ Nano-Electronics Centre, Advanced Technology Institute, School of Electronics and Physical Sciences, \\ University of Surrey, Guildford GU2 7XH, United Kingdom
}

(Received 31 August 2005; accepted 11 January 2006; published online 21 February 2006)

\begin{abstract}
Pulsed-laser $(248 \mathrm{~nm})$ irradiation of $\mathrm{Ag}$ thin films was employed to produce nanostructured $\mathrm{Ag} / \mathrm{SiO}_{2}$ substrates. By tailoring the laser fluence, it was possible to controllably adjust the mean diameter of the resultant near-spherical Ag droplets. Thin films of tetrahedral amorphous carbon (ta-C) were subsequently deposited onto the nanostructured substrates. Visible Raman measurements were performed on the ta-C films, where it was observed that the intensity of the Raman signal was increased by nearly two orders of magnitude, when compared with ta-C films grown on nonstructured substrates. The use of laser annealing as a method of preparing substrates, at low macroscopic temperatures, for surface-enhanced Raman spectroscopy on subnanometer-thick films is discussed. () 2006 American Institute of Physics. [DOI: 10.1063/1.2178387]
\end{abstract}

The number of potential applications for nanoparticles, including protein detection, ${ }^{1}$ catalysis, ${ }^{2}$ and gas sensing, ${ }^{3}$ is rapidly growing because of their unique electronic structure and large surface areas. Metal nanoparticles and other metal nanostructures are the basic building blocks in photonics research ${ }^{4}$ and it is expected that magnetic nanoparticles will have a dramatic impact on high density magnetic recording. ${ }^{5}$ The synthesis of nanosized noble metal particles has become an important branch of research due to their interesting optical, ${ }^{6}$ chemical, ${ }^{7}$ and electronic ${ }^{8}$ properties. For example, $\mathrm{Ag}$ nanoparticles are of particular interest due to their surface-enhanced Raman effect, ${ }^{9}$ which can increase the sensitivity of Raman measurements by orders of magnitude, even allowing single molecule detection. Typically nanostructured silver substrates for surface-enhanced Raman spectroscopy (SERS) are prepared by chemical means from citrate reduced Ag colloids. ${ }^{10}$

In this letter we introduce a new method for producing supported arrays of Ag nanoparticles, over large area, and at low macroscopic temperatures. Once produced, we investigate the effectiveness of these arrays as substrates for performing SERS, by probing how the carbon bonding configuration varies with film thickness for ultrathin amorphous carbon $^{11}(a-\mathrm{C})$ coatings. High ratios of $s p^{3}$ to $s p^{2}$ bonded carbon produces hard, wear resistant, tetrahedral amorphous carbon (ta-C) films, which are of considerable interest for many applications including coating of hard disk platters. For such applications, very thin ta-C coatings are required, but how thin can they be deposited whilst still retaining their diamond-like properties?

$\mathrm{Ag}$ thin films were deposited by pulsed laser ablation ${ }^{12}$ (PLA) of a 99.9\% pure Ag foil. A Lambda-Physik LPX 210i excimer laser, producing $25 \mathrm{~ns}$ pulses at $248 \mathrm{~nm}$, was used for the ablation. Thin films were producing using a laser fluence of $\sim 10 \mathrm{~J} / \mathrm{cm}^{2}$. The growth chamber was evacuated using a turbo pump to $9 \times 10^{-8}$ Torr. The substrates used were $\mathrm{SiO}_{2} / \mathrm{Si}$ with the thermal oxide having a thickness of $235 \mathrm{~nm}$. The target to substrate distance was $6 \mathrm{~cm}$ for all depositions. Ag films were deposited with a thickness from

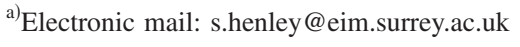

5 to $20 \mathrm{~nm}$, the growth rate being calibrated by profilometry. The Ag films were excimer laser nanostructured (ELN) using the same excimer laser as employed for the deposition, by diverting the optical path and passing it through a homogeniser. This technique is discussed in more detail elsewhere. ${ }^{13}$ The films were transferred to a separate vacuum stage (typical working pressure $10^{-4}$ Torr) where the whole sample could be laser annealed by translating the stage. The typical translation rate was $1 \mathrm{~mm} / \mathrm{s}$ with a laser repetition rate of $10 \mathrm{~Hz}$. This method has the advantage, compared to traditional thermal annealing, that the majority of the laser energy is absorbed in the thin metal film, such that the substrate remains at low macroscopic temperatures. Postannealing the structure of the films was investigated in a Hitachi S4000 field emission gun scanning electron microscope (SEM).

After morphological characterization of the substrates, their suitability for performing SERS measurements was investigated by performing Raman spectroscopy measurements using a Renishaw Ramanscope with $514 \mathrm{~nm}$ excitation. Thin ta-C films were deposited onto the nanostructured $\mathrm{Ag}$ and the untreated $\mathrm{Ag}$ films, by PLA in vacuum of a $99.99 \%$ pure pyrolytic graphite target. Again, a fluence of $\sim 10 \mathrm{~J} / \mathrm{cm}^{2}$ was used for the ablation. For comparison ta-C films were also deposited straight onto the $\mathrm{SiO}_{2}$ substrates. The ta-C film thickness was varied from 0.5 to $15 \mathrm{~nm}$. The Raman spectra of these ultrathin diamondlike carbon (DLC) film were obtained using $1 \%$ of the full laser output to minimise any effects of laser heating on the bonding configurations in the samples and to avoid saturating the detector during SERS measurements. It should be noted, however, that no observable changes in the spectral shape was observed at higher laser powers.

Figure 1 shows SEM images of a $15 \mathrm{~nm}$ thick Ag film after it was ELN at different fluences. At low fluences [see Fig. 1(a)] the film is observed to perforate in some areas and breakup into large droplets. As the laser fluence is increased the whole irradiated area breaks up into near spherical droplets with diameters of around $150 \mathrm{~nm}$ [see Fig. 1(b)]. A similar breakup into droplets has been observed for the ELN of $\mathrm{Ni}$ thin film. ${ }^{13}$ It should be noted that the liquid $\mathrm{Ag}$ is not expected to wet the oxide surface. Well above the threshold 


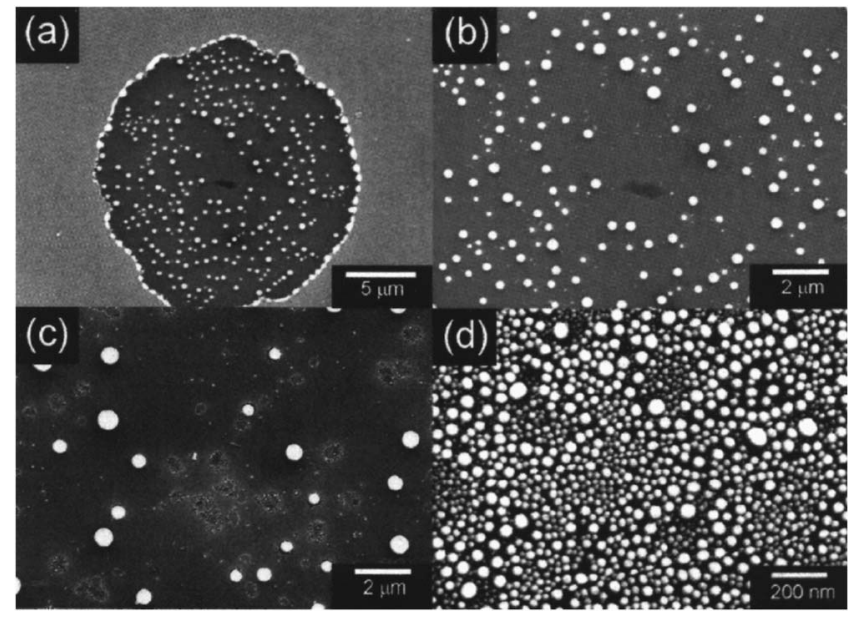

FIG. 1. SEM images of ELN $15 \mathrm{~nm}$ thick Ag films. The fluences used were (a) 100, (b) 150 , (c) 300 , and (d) $400 \mathrm{~mJ} / \mathrm{cm}^{2}$.

for breakup [see Fig. 1(c)] smaller droplets are observed around the larger ones. At the highest fluences studied [see Fig. 1(d)] all the large droplets are gone and are replaced by a high density of much smaller droplets. Statistical analysis of the $\mathrm{Ag}$ nanoparticle diameter distributions produced by the ELN of Ag thin films, at fluences of $150-600 \mathrm{~mJ} / \mathrm{cm}^{2}$, was performed. From these distributions a shift of the distribution from a mean diameter of $144 \pm 6 \mathrm{~nm}$ down to $21.6 \pm 0.5 \mathrm{~nm}$ was observed. The larger diameter droplets, produced by hydrodynamic instabilities of the liquid metal on the oxide surface, appear to be fragmenting as they boil to produce many more smaller droplets. If the film is boiling and fragmenting, metal atoms should be lost into the vacuum during the ELN. Figure 2 shows two SEM images of Ag films ELN at $400 \mathrm{~mJ} / \mathrm{cm}^{2}$. The initial film thickness was (a) 5 and (b) $20 \mathrm{~nm}$. The size and distribution of droplets is similar is both cases, indicating a considerable fraction of the metal film must be being boiled away from the thicker film, and indeed, a coating of ablated metal is observed on the window of the ELN chamber after processing at these higher fluences.

After laser processing of $15 \mathrm{~nm}$ thick $\mathrm{Ag}$ films at the higher fluence $\left(\sim 400 \mathrm{~mJ} / \mathrm{cm}^{2}\right)$ the film were converted into supported arrays of Ag nanoparticles. These arrays were then coated with thin ta-C coatings, of different thicknesses. The suitability of these substrates for performing SERS was evaluated by taking visible Raman spectra of the ta-C films. However, before making these measurements the Raman spectra of the thin films deposited on bare $\mathrm{SiO}_{2}$ and unmodified Ag thin films, was measured.

The inset in Fig. 3(a) shows a visible Raman spectrum for the $15 \mathrm{~nm}$ thick DLC film deposited onto $\mathrm{SiO}_{2} / \mathrm{Si}$, taken at a higher laser power and with a longer collection time than the other spectra shown in Fig. 3. Typically the visible Raman spectra of $a$-C films are characterised as a combination of two bands, typically labeled the $D$ and $G$ band and are attributed to the breathing mode of aromatic rings and inplane bond stretching of pairs of $s p^{2}$ bonded $\mathrm{C}$ atoms, respectively. ${ }^{14}$ For $514 \mathrm{~nm}$ excitation, the $D$ band is typically located at $1335 \mathrm{~cm}^{-1}$ and the $G$ band in the range $1580 \pm 20 \mathrm{~cm}^{-1}$. In order to characterize the films, the Raman spectra were fitted using a Breit-Wigner-Fano line for the $G$ band and a Lorentzian for the $D$ band. ${ }^{14}$ A linear background due to photoluminescence was assumed. The solid line shown in the inset of Fig. 3(a) is a fit to the spectrum using a Breit-Wigner-Fano line for the $G$ band. No $D$ band contribution was required to fit the spectrum, which is characteristic for as deposited ta-C films. ${ }^{14}$

Figure 3 shows Raman spectra of ta-C films with thickness (a) 15, (b) 2, and (c) $0.5 \mathrm{~nm}$ deposited onto different substrates. Spectra (i), (iv), and (vii) are from films deposited on ELN Ag, spectra (ii) and (v) on Ag thin films and spectra (iii), (vi), and (viii) on "bare" $\mathrm{SiO}_{2}$ substrates. The spectral intensity measured from the thinner two ta-C films deposited on bare $\mathrm{SiO}_{2}$ are too weak to resolve the $D$ and $G$ bands. However, a dramatic enhancement of the signal is observed in all cases for the films deposited on the Ag films and the ELN Ag substrates. The enhancement factor (defined as the ratio of the integrated intensities of the Raman signal between 1000 and $1800 \mathrm{~cm}^{-1}$, after subtraction of the linear background) is significantly greater for the ELN substrates, compared with the Ag films, and decreases as the thickness of the ta-C coating increases. The maximum enhancement observed, was by a factor of $\sim 80$, for a $1 \mathrm{~nm}$ ta-C coating deposited on ELN Ag.

Finally, we consider the form of the Raman spectra obtained on the ELN Ag substrates, where in comparison to the $15 \mathrm{~nm}$ thick film on $\mathrm{SiO}_{2}$ [inset Fig. 3(a)], the $D$ band contribution is more pronounced. The ratio of the intensities of the $D$ and $G$ bands increases, as the thickness of the C coating is decreased. This change in relative intensities of the bands is typically associated with increased ordering of the $s p^{2}$ bonded $\mathrm{C}$ phase, going from $s p^{2}$ dimers in $s p^{3}$ matrix to rings and chains of $s p^{2}$ bonded $\mathrm{C}$ atoms. ${ }^{14}$ It is known that stress relaxation in ta-C forms a $s p^{2}$ rich surface layer. As these coatings were all deposited by PLA at the same laser fluence, but with different thickness, the changes in the spectra must be due to the relative contribution, to the total, of the Raman signal from the bulk of the film. For thinner films, the SER contribution from layers at the top or bottom surfaces becomes more important due to the top surface getting closer to the Ag substrate and the signal from the bulk becoming smaller relative to the SER signal from the bottom surface. The spectra indicate that, at these surfaces, the $\mathrm{C}$ bonding configuration is different from the bulk of the ta- $\mathrm{C}$

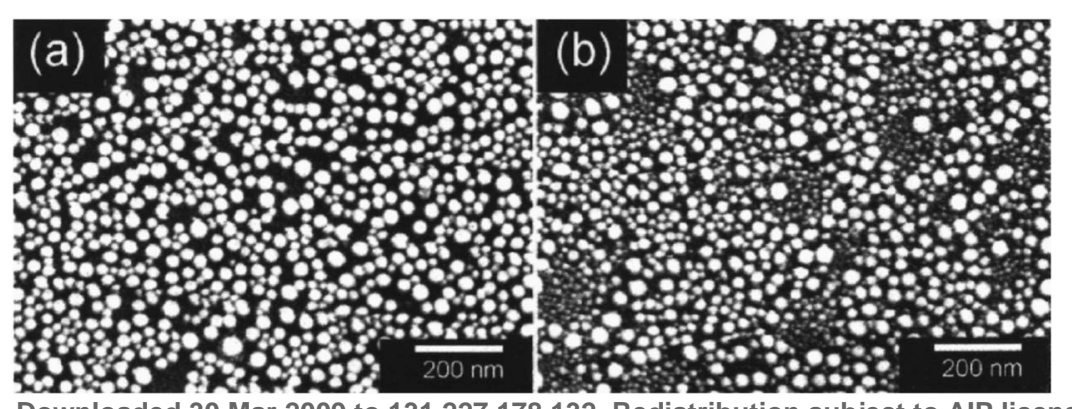

FIG. 2. SEM images Ag films ELN at $400 \mathrm{~mJ} / \mathrm{cm}^{2}$. Initial film thickness was (a) 5 and (b) $20 \mathrm{~nm}$. 


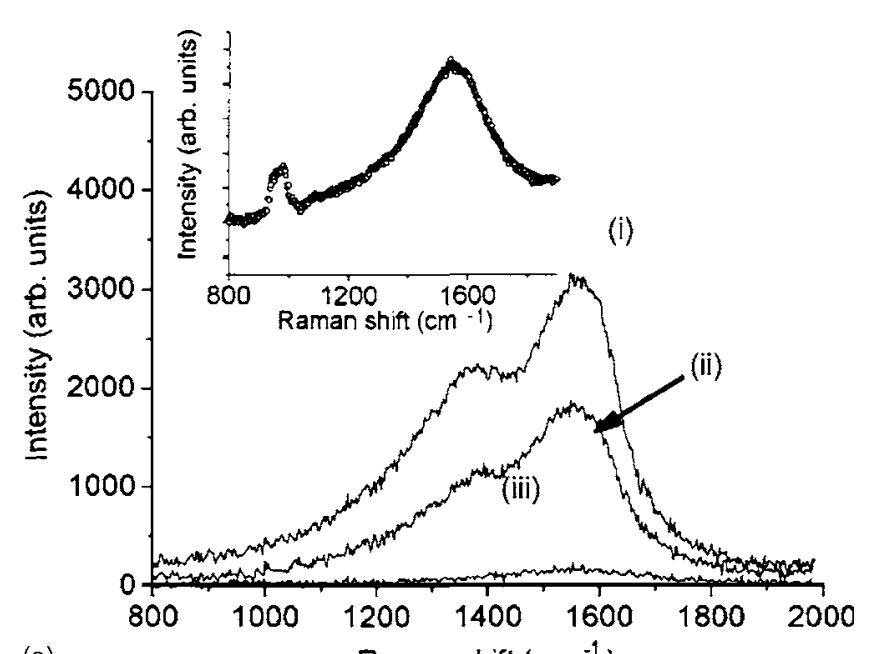

(a) Raman shift $\left(\mathrm{cm}^{-1}\right)$

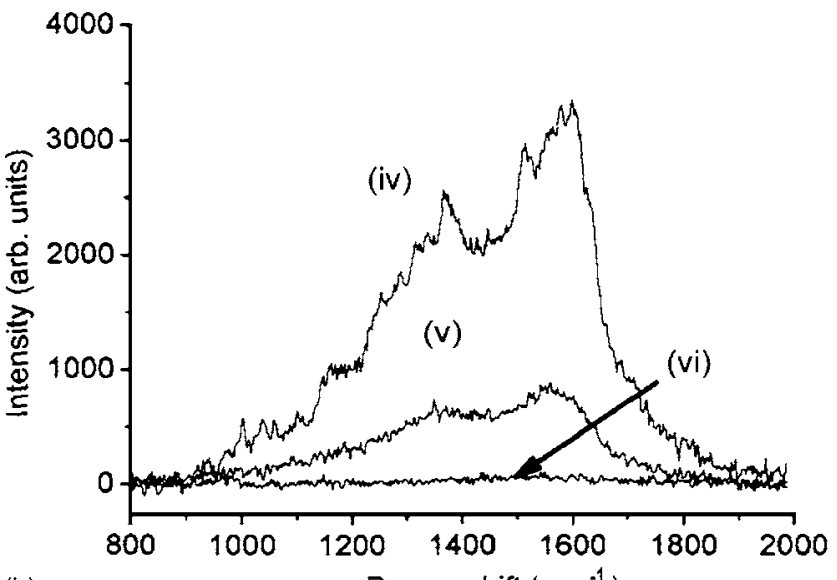

(b)

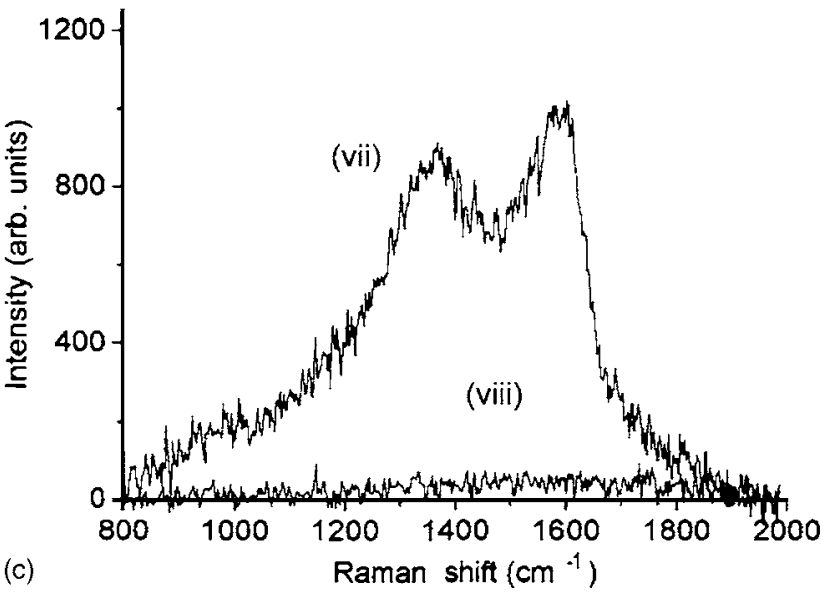

FIG. 3. Raman spectra of ta-C films with thickness (a) 15, (b) 2, and (c) $0.5 \mathrm{~nm}$. Spectra (i), (iv), and (vii) from films deposited on ELN Ag, (ii) and (v) $\mathrm{Ag}$ thin films and (iii), (vi), and (viii) $\mathrm{SiO}_{2}$ substrates. The enhancement on the $\mathrm{Ag}$ substrates is evident. Inset in (a) is the Raman spectrum of the $15 \mathrm{~nm}$ thick sample on $\mathrm{SiO}_{2}$ taken at a higher laser power.

film. These interface layers becoming a greater component of the whole film as the thickness is decreased. As the bonding configuration is different in these layers, their hardness is likely to be reduced, an observation that has implications for the deposition of scratch-resistant thin coatings for hard disk platters.
In conclusion, we have demonstrated that UV pulsed laser irradiation of silver thin films can be employed to produce nanostructured $\mathrm{Ag} / \mathrm{SiO}_{2}$ substrates over large area, at low macroscopic temperatures, suitable for performing SERS. This approach has the potential for broader usage as the ELN mechanism can be applied to wide range of other suitable metals, including $\mathrm{Au}, \mathrm{Pt}$, and $\mathrm{Ni}^{15}$ This nanostructuring process could also have important applications in catalysis and photonics. After laser nanostructuring, the films consisted of discrete nanometer-scale near-spherical droplets. These substrates were used to perform SERS measurements on ultrathin ta-C coatings. An increase in the Raman signal of nearly two orders of magnitude was observed when using these nanostructured substrates, allowing the detection of subnanometer thicknesses of amorphous carbon. It was demonstrated that the carbon bonding configuration varied with film thickness, and the existence of softer interface layers at the top and/or bottom surfaces of the films is predicted, which has implications for the deposition of ultrathin scratch-resistant coatings. The ELN approach has advantages over traditional "wet-chemical" methods for producing nanostructured metal surfaces. The process is compatible with vacuum microelectronics fabrication processes and can be easily patterned. Also the process can be used to produce nanoislands of different metals, on the same substrate, with diameters that are readily controlled by just the laser parameters. All this means ELN will be important for future highdensity lab-on-chip SERS applications where many different tests can be run on the same analyte.

The authors would like to thank the EPSRC Portfolio Grant and the Carbon Based Electronic Programme for funding the project. S.J.H. is grateful to J. Filik, G. M. Fuge, and P. W. May from the School of Chemistry at the University of Bristol for assistance in performing the Raman measurements.

${ }^{1}$ J. M. Nam and C. C. Thaxton, Science 301, 1884 (2003).

${ }^{2}$ A. T. Bell, Science 299, 688 (2003).

${ }^{3}$ M. K. Kennedy, F. E. Kruis, H. Fissan, B. R. Mehta, S. Stappert, and G. Dumpich, J. Appl. Phys. 93, 551 (2003).

${ }^{4}$ T. Goodson, O. Varnavski, and Y. Wang, Int. Rev. Phys. Chem. 23, 109 (2004).

${ }^{5}$ H. Kodama, S. Monose, N. Ihara, T. Uzumaki, and A. Tanaka, Appl. Phys. Lett. 83, 5253 (2003).

${ }^{6}$ W. H. Weber and G. W. Ford, Phys. Rev. B 70, 125429 (2004).

${ }^{7}$ P. Waszczuk, T. M. Barnard, C. Rice, R. I. Masel, and A. Wieckowski, Electrochem. Commun. 4, 599 (2003).

${ }^{8}$ S. Link and M. A. El-Sayed, J. Phys. Chem. B 103, 8410 (1999).

${ }^{9}$ Y. Lu, G. L. Liu, and L. P. Lee, Nano Lett. 5, 5 (2005).

${ }^{10}$ P. C. Lee and D. J. Meisel, J. Phys. Chem. 86, 3391 (1982).

${ }^{11}$ S. R. P. Silva, J. D. Carey, R. U. A. Khan, E. G. Gernster, and J. V. Anguita, in Handbook of Thin Film Materials, edited by H. S. Nalwa (Academic, New York, 2002).

${ }^{12}$ M. N. R. Ashfold, F. Claessyens, G. Fuge, and S. J. Henley, Chem. Soc. Rev. 33, 23 (2004).

${ }^{13}$ S. J. Henley, C. H. P. Poa, A. A. D. T. Adikaari, C. E. Giusca, J. D. Carey, and S. R. P. Silva, Appl. Phys. Lett. 84, 4035 (2004).

${ }^{14}$ A. C. Ferrari and J. Robertson, Phys. Rev. B 61, 14095 (2000).

${ }^{15}$ S. J. Henley, J. D. Carey, and S. R. P. Silva, Phys. Rev. B 72, 195408 (2005). 